\title{
Usability Engineering of a Radiological Workstation for Mobile Devices: An Experience Report
}

\author{
Pedro van Rooij Costa ${ }^{1}$, Aldo von Wangenheim ${ }^{1}$, Christiane G. von Wangenheim ${ }^{1}$, \\ Andrei de Souza Inácio ${ }^{1}$, Douglas D. J. de Macedo ${ }^{2}$ \\ ${ }^{1}$ Department of Informatics and Statistics (INE) \\ ${ }^{2}$ Department of Information Science (CIN) \\ Universidade Federal de Santa Catarina (UFSC) \\ Caixa Postal 476 - 88.010-970 - Florianópolis - SC - Brazil \\ \{pedro, andrei\}@incod.ufsc.br, \{aldo.vw, c.wangenheim, douglas.macedo\}@ufsc.br
}

\begin{abstract}
Medical imaging is commonly used to diagnose many emergent conditions as well as plan treatments. Digital images can be visualized on almost any computing device that has a screen. These days most devices used to visualize digital images are still desktops. Despite that, the world that surrounds us is evolving to a more mobile environment, therefore, mobile devices are increasingly being incorporated onto Picture Archiving and Communication Systems (PACS). In this paper we describe the usability engineering of a new medical image viewer for iPads as a Teleradiology tool for evaluating CTs, X-Rays, Ultrasounds, etc. First we gathered requirements, after we created a series of prototypes and finally the development of the actual application. The experience resulted in insights into how to develop an appropriate viewer for the specific target end user group. Our results suggest that emergency conditions commonly encountered on CT and MRI can be diagnosed using tablet computers with good agreement with dedicated PACS workstations, accordingly to ACR (American College of Radiology). Shortcomings in application design should be addressed if the potential of tablet computers for mobile Teleradiology is to be fully realized.
\end{abstract}

\section{Introduction}

The use of mobile devices such as smartphones or tablets is revolutionizing the way we communicate and interact [Kim 2011]. This has also created opportunities for Teleradiology applications that allow an on-the-go interpretation of medical images as opposed to the use of an office-based workstation, optimizing referring physicians' access to images and reports from anywhere, anytime. With growing demands on radiology services worldwide, there are many scenarios in which such mobile teleradiology devices can be used to support rapid medical and imaging decision making, for instance, in an emergency situation when doctors are away from the hospital. Enabling rapid expert review from an experienced radiologist anywhere anytime facilitates interventions that [John 2012] allow safe and timely communication of important radiological findings to the clinician that may affect the patient's treatment, while at the same time, removing restrictions in physical location [Avitzur 2010]. Moreover, by removing geographical and temporal boundaries, these technologies may reduce economic disparities, lessen healthcare costs, and promote more personalized healthcare [Weiss 2010].

Thus, mobile radiological diagnosis support employing wireless communication devices can be a desirable extension of imaging services, particularly as an additional feature of an existing Picture Archive and Communication System (PACS), normally processing DICOM 
images, which are a standard format associated with PACS. In this context, portable tablet computers, which have a large high-resolution display and touchscreen interface, may have potential to successfully allow the remote image review of radiological studies. Among current tablet computers, Apple's iPad is one of the most prominent hand-held devices, with diverse health care applications in the teleradiology field, also holding the largest market share of this kind of device and a high adoption rate among physicians.

Compared to medical workstations, tablets have several limitations: small screen size, inherent low resolution of display, low contrast ratio, poor connectivity, slow data transfer, security issues, and minimal inherent memory [Avitzur 2010]. Additionally, they most probably will not be used in an environment with controlled ambient lighting, as is expected from radiological workstations used in accordance to recommendations such as AAPM TG-18 or norms such as DIN V 6868-57 [Choudhri 2012]. Therefore, most mobile DICOM-viewing applications come with a disclaimer of "not for primary diagnosis", and there is a paucity of published literature documenting the accuracy of primary radiological diagnosis made on tablet computers [Wasserman 2010]. Nevertheless, there are several studies that indicate the potential usefulness of the iPad for radiological diagnosis of multi-image CT and MRI studies in afterhours conditions, where a diagnosing physician can view the images from home, another office, his car or any other place, using a tablet computer and then send his diagnosis and treatment instructions. Recently, the US Food and Drug Administration has also issued clearance for the use of a specific software application (Mobile MIM, [Chen 2009]) on the iPhone and iPad for viewing and diagnosis of CT, MR imaging, and nuclear medicine images where access to primary-class displays is an issue, even though it accesses DICOM images it has a drawback of needing to have specific DICOM servers also provided by Mobile MIM.

Another issue in this context is the required degree of software quality, as these applications often fall short on usability. Medical software manufacturers have been providing applications with Graphic User Interfaces (GUI) that are not necessarily compliant with standard usability criteria [Drnasin 2010]. On the other hand, there are several DICOM viewers available to download and purchase that seem to be accepted such as OsirixMD, MobileMIM, VueMe and ResolutionMD.

The need to ensure that medical applications and devices are designed, manufactured and also used in a way that does not lead to unnecessary risks to patients and users is not new. We need to observe specific recommendations from devices until the conditions about the environment in radiological images [Choudhri 2012]. As the design for patient safety initiatives play an increasing role in public health policy, it becomes necessary to consider ergonomic design in the essential requirements.

In this way, usability is "the extent to which a product can be used by specified users to achieve specified goals with effectiveness, efficiency and satisfaction in a specified context of use" [ISO 2002]. In essence, a system with good usability is easy to use and effective. It is intuitive, forgiving of mistakes and allows one to perform necessary tasks quickly, efficiently and with a minimum of mental effort. Usability represents an important, although often overlooked, factor that impacts the adoption and meaningful use of PACS and mobile DICOM viewers. In mobile applications, usability is really important because it is a different environment than traditional desktop systems [Yen 2011]. If an application cannot be correctly usable, the accomplishment of simple tasks becomes more difficult and error-prone.

Being mobile devices, tablets and smartphones can be used in different places and contexts, e.g. on the move anywhere and anytime. Therefore, many common desktop design patterns and usability principles do not apply in a mobile environment [Siau 2004]. Small screens and limited input methods create extra challenges for interaction design. For example, small screen size implies less visible content and options that can make almost all interactions with such devices harder [Yen 2011]. It is therefore important how health information is displayed for stakeholders without losing important parts or how to design navigation so that people will not have difficulties to access the required information. Navigation mechanisms 
(including searching and browsing) for applications need to be designed for the small screen, and direct data entry carried out via touch and multi-touch interactions. For example, it is important to provide a logical path for the user to follow, making a navigation control consistent and obvious. In addition, in the mobile domain, users will find it difficult if an application relies heavily on text input, so it is important to use alternatives, for instance table views or dropdown menus in order to minimize the effort required for user input [Choi 2012].

In this paper we describe the development of a DICOM viewer application for the iPad, created to be used in the context of a public statewide telemedicine network, the STT/SC in Southern Brazil [STT 2016]. This application was explicitly developed accordingly to usability guidelines and through context analysis and prototypes made before the creation of the actual application. After the application was developed, we performed usability tests with potential users of the visualizer in order to evaluate the application, in order to validate applicability of this DICOM visualizer for the iPad as an auxiliary device in the process of performing and issuing medical reports in a Teleradiology environment. In this study we analyzed if the application is well developed from the users' point of view and if all the required tools are present and easy to use, following all common guidelines of usability for mobile devices and medical image viewers.

The application development started with studies of guidelines and usability for mobile applications, especially image visualizers. Following this study we gathered user requirements interviewing users of our Telemedicine network, and then started to prototype the interfaces using the knowledge gathered through the study and the requirements. After that, the actual application was developed following usability and software engineering. With the application ready we did evaluations with potential users and analyzed all the data and results gathered.

\section{Related Work}

So far few specific usability guidelines for mobile medical apps have been proposed. Nielsen (1994), on the other side, proposes a number of generic guidelines for increasing usability of mobile devices as well as touch-screen application usability. So far, there are basically design guidelines created by platform providers and/or handset manufacturers, e.g. iOS design guidelines, UI guidelines for Android, and UI guidelines for Windows Mobile. There are also evaluations of Nielsen heuristics (1994) by Chan (2011) and Yen (2011).

Especially research regarding the usability of a medical image viewer is very sparse. One exception is the work by Chen (2009), which, however, does not focus on mobile devices. On the other hand, Cannella (2009) only focus on technical issues regarding the automatic creation of interfaces.

Therefore, we present in this article an experience report on the usability engineering of the development of a mobile DICOM viewer application for the iPad for the visualization of radiological images in the context of a large-scale Telemedicine network. The application is being developed and integrated into the STT/SC - Santa Catarina State Integrated Telemedicine and Telehealth System - a public asynchronous web-based telemedicine network in the state of Santa Catarina/Brazil [STT 2016], that performs store-and-forward telemedicine and findings reports delivery in the fields of: clinical laboratory analysis, radiology (MR, US, CT, SPECT, bone densitometry), endoscopy and colonoscopy, and ECG and tele dermatology, besides asynchronous emergency assessment, mainly on trauma cases. Today, the network interconnects more than 400 hospitals and primary health care facilities in 295 cities, performing, on average, more than 70,000 examinations/month.

\section{Material and Methods}

We performed this case study through a systematic development of a DICOM viewer to answer the questions raised previously. The development stages are shown in Figure 1. Figure 1 shows all the stages of the application development; firstly there is a literature analysis, after, prototyping of user interface, application development and finally the evaluation of the 
application and interfaces created.

The research was executed as a cooperation between the GQS - Software Quality Group and the LabTelemed - Telemedicine Laboratory of the Brazilian Institute for Digital Convergence (INCoD) at the Federal University of Santa Catarina and the Santa Catarina State Health Department - SES/SC, involving researchers from the area of usability engineering, design and software engineering, as well as from the area of telemedicine.

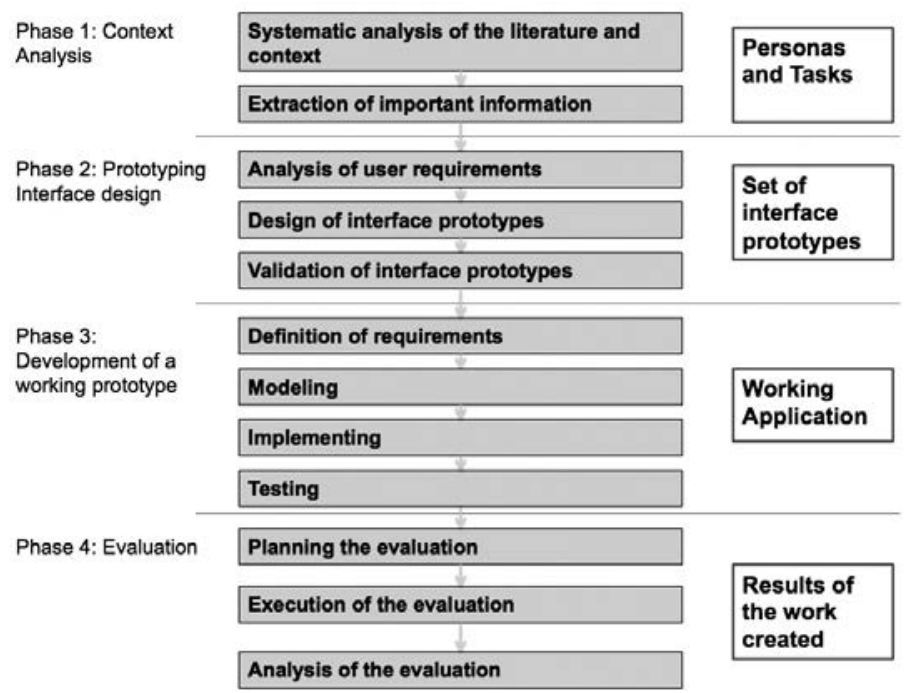

Figure.1. Stages of the application development

In order to achieve high usability, interface design was a priority from the very beginning of the project. Design input, design verification and design validation were conducted throughout the design and development of the medical application. Design input calls for manufacturers to establish and maintain procedures that ensure design requirements are appropriate and address the intended use of the device, user needs and patient needs.

A specific iterative design process model is presented by ISO 9241-210. This "humancentered design" approach focuses on the needs of the future user in order to develop a usable human-machine-interface. Principles are, among others, an explicit understanding and integration of users, their tasks and realistic environment, iterative processes and multidisciplinary skills and perspectives within the design teams.

\subsection{Phases}

In this phase (Phase 1 - Context Analysis) General requirements were obtained from the ACR standard for teleradiology. It states that a radiological viewer must be capable, among other features, to select image sequences, accurately associate the patient and study, adjust window and level, pan and zoom image, rotate or flip the images provided, calculate and display accurate linear measurements and pixel value determinations in appropriate values for the modality (e.g., Hounsfield units for CT images), and display prior image compression ratio, processing or cropping.

In order to elicit characteristics, preferences and needs of the target users of the application, we performed a series of interviews with radiologists and domain experts - all professionals working for the public telemedicine system in Santa Catarina/Brazil and familiar with the concept of Teleradiology. Based on the analysis of the collected data, the users have been characterized and illustrated through the definition of personas. Based on the analysis results, the following usability requirements have been defined: 
-UR1. All commonly used functions (measuring, zoom, windowing and leveling) have to be accessible by at most 2 clicks;

-UR2. The use of two fingers simultaneously should be possible;

-UR3. Zoom should be done using the pincer movement;

-UR4. The task "visualize and interpret exams" should be completed in at least $70 \%$ cases;

-UR5. The task "visualize and interpret exams" should take less than 10 minutes;

-UR6. The degree of user satisfaction should be at least 60 points using the SUS (Unified Health System in Portuguese language) - satisfaction questionnaire.

During the next phase (Phase 2 - Interface Design Prototyping), we produced design solutions, trying to meet user requirements as well as possible. This is done by iteratively prototyping designs starting with sketching low-fi paper prototypes. These first prototypes have been evaluated through cognitive walkthroughs. Based on the feedback, we improved the design, e.g. reducing the size of the inferior toolbar and re-positioning of the return button. For this prototyping iteration we used a wireframe tool (Figure 2). Based on the feedback, we included an additional logon screen for the login. Another issue we observed was the need for a more direct and quick visualization of images rather than first presenting the patient and examination data, which the physician may have already accessed beforehand. Therefore, we eliminated in the sequence of screens the screen that visualized patient and examination data only. Instead, after selecting a patient and examination, the system directly starts to visualize images - allowing the physician, if necessary, to push down an overlay with the patient and examination information.

During the next phase (Phase 3 - Development of a Prototype), a working prototype for studying the interaction with end users was created. Here, we concentrated on the Hi-Fi prototype and adapted the choice of software tools to technological requirements. We initially performed a requirement analysis, covering the identification of functional and non-functional requirements, including the usability requirements as identified in the context analysis. Using this analysis as a starting point, the modeling of the MVC (Model-View-Controller) architecture was performed. Immediately after this, a class diagram was created with the objective to define the application. After this first step, the app interfaces where created and integrated with the model using the previously created controllers as interfaces between them. All of these functionalities were created in Mac OS using XCode as the app development program.

In next phase (Phase 4) his evaluation had the objective to validate the usability of the user interface we designed and developed and the functionalities implemented from the viewpoint of the doctors who perform Teleradiology examination reports for the STT/SC system. The objectives of the evaluation are:

-Analyze the effectiveness, efficiency and satisfaction of the interfaces and functionalities;

-Analyze if the functionalities developed are enough;

-Validate the application as an aid on the process of creating an examination report;

-Identify the strong and weak points of the design created with the goal of improving it and

-Verify if the requirements were met.

To simulate the situation during exam analysis, the participants had as much time as they desired to review the data set using the working prototype. The test was performed on an iPad 2, running iOS 6.1.4 as the operating system. Observers were allowed to use all functions of the DICOM viewer on both systems, including zooming, panning, and control of radiological window width and level.

At the start of each session, the study procedures were explained to each participant and 
written assent was obtained. The participants were then asked to fill out a short demographic questionnaire. Each participant performed the defined task on images related with their expertise. Participants were asked to think aloud and to verbalize the steps taken to carry out each task as they used the devices. Participants' interactions with the devices were video and audio-recorded. Data collection was performed via video recording, post-test questionnaire, and unstructured interview. Equipment used in the usability test to collect all data were two cameras, one notebook and a support to fix the iPad and the cameras (figure 3). See the video on our website: http://site.telemedicina.ufsc.br/?page_id=410.
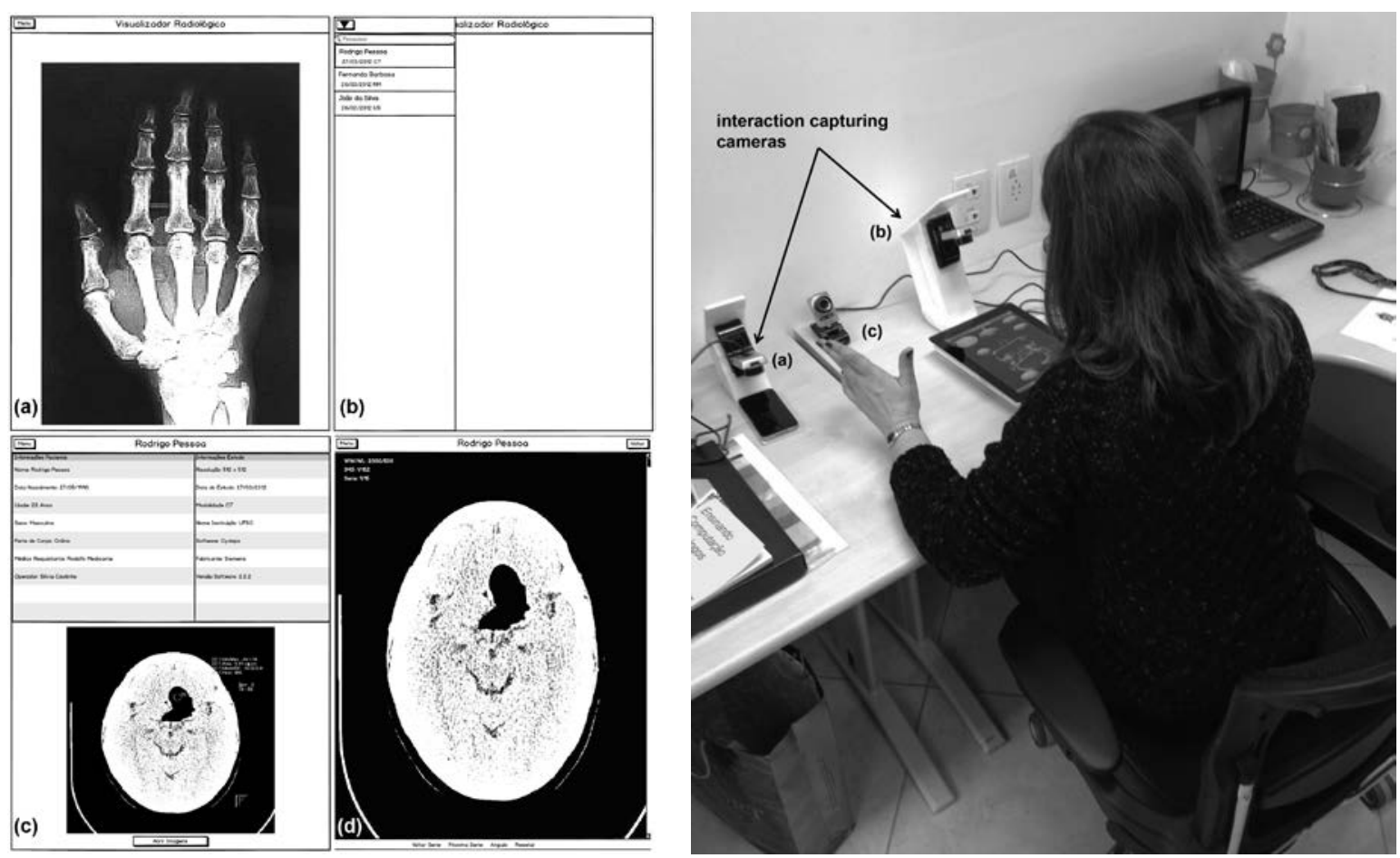

Figure.2. Left: Screen mockups of the application created with: (a) start screen, (b) patient list, (c) patient and study information and (d) study visualization. Right: Usability test set showing an interaction situation on the iPad tablet. Interactions on the device were captured through specially developed camera mounts for the cellphone (a) and the tablet (b). User attention, gazing and eye movements were captured through a second camera (c).

The notebook works as a server to capture images from both cameras simultaneously. One camera is directed to the participant's face to capture facial expressions, gazing and eye movements (see fig.3 (c)). The second camera is directed to the mobile device screen to capture the participant's interaction with the device (see fig.3 (a) and (b)). The audio is captured through the microphone of both cameras. During each session, one member of the study team moderated the usability test. After performing the task participants completed a post-test questionnaire based on SUS satisfaction questionnaire with additional questions with respect to the evaluation objectives. Demographic data of the participants is given in the table I below.

TABLE I

\begin{tabular}{llll}
\multicolumn{4}{c}{ DEMOGRAPHIC DATA } \\
\hline Participant & Specialty & STT User & Has an iPad \\
\hline 1 & Radiology & Yes & Yes \\
2 & Stomatology & Yes & Yes \\
3 & Neurosurgery & Yes & Yes \\
4 & Obstetrics & Yes & Yes \\
5 & Radiology & Yes & Yes \\
6 & Radiology & Yes & Yes \\
\hline \hline
\end{tabular}


Each item in the SUS questionnaire is measured on a 7-point Likert scale. Responses range from 1 (strongly agree) to 7 (strongly disagree). The other part of the questionnaire is made of specific question to evaluate all the other objectives that are not contemplated by the SUS questionnaire. All the questions have to be answered with "yes" or "no" and if it has a negative answer the participant should explain why.

The tests were taken between 6 and 10 of may of 2013. They were taken in the Governador Celso Ramos Hospital (HGCR), children's hospital and INE-CTC at UFSC (Universidade Federal de Santa Catarina). In total there were 5 participants, all doctors of the SUS-SC (Unified health system of the Santa Catarina state). These participants are representatives of the users of the application created. The participants were chosen through phone contact with the help from the technical support of telemedicine in Santa Catarina.

The data were analyzed through observation of the participants during the test and manual video analysis (see Figure 3 (a) and (b)). On the videos, errors were manually identified when a participant executed an incorrect action in carrying out task steps. When the task was completed, it was coded as meeting a task goal. With the use of questionnaires, it was also possible to detect more specific problems and acknowledgments about the use of the application that the video couldn't detect. The answers were analyzed and used to form data tables making it easier to compare all the results and get an overall impression of the performance of the application.

According to the ACR (American College of Radiology) images of computerized tomography $(\mathrm{CT})$, magnetic resonance (MR) and ultrasound (US) require a monitor with at least $512 \times 512$ pixels and 8 pixels of depth (providing 256 shades of gray) so that there is no loss of processing and image manipulation [Drnasin 2010]. The iPad provides a screen resolution of $2048 \times 1536$ pixels and also enough space for visualization for almost all radiological images, as seen on table II, which shows information about the typical resolution, average quantity of images per exam and the space occupied in megabytes for common modalities of imaging study. For the study we randomly selected CT and MR imaging cases from the STT/SC database. CT cases were selected from studies of different anatomies, including dental and torso.

TABLE II

AVERAGE SiZE OF IMAGES PER MODALITy [DRNASIN 2010]

\begin{tabular}{llll}
\hline Modality & Image Size $(\mathrm{px})$ & $\begin{array}{l}\text { Images per } \\
\text { Study }\end{array}$ & Space $(\mathrm{MB})$ \\
\hline Tomography & $512 \times 512$ & $40-3000$ & $20+$ \\
Ultrasound & $512 \times 512$ & $15-240$ & $5-60$ \\
$\begin{array}{l}\text { Magnetic } \\
\text { Resonance }\end{array}$ & $256 \times 256$ & $50-2000$ & $8+$ \\
\hline \hline
\end{tabular}

\section{Experimental Results}

Having the interface prototypes defined, the process of implementing the software was was performed.

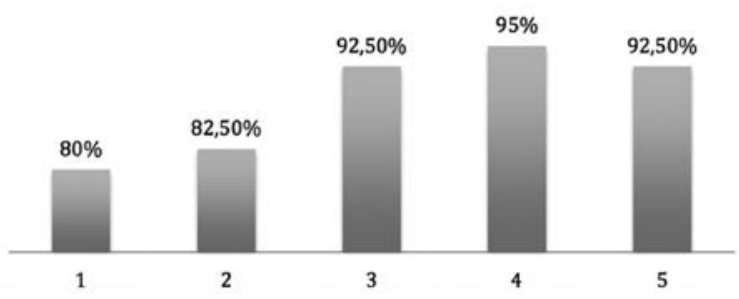

Fig.4.SUS evaluation results per participant. Scores have a minimum value of $0 \%$ and maximum value of $100 \%$. 
To achieve the goals defined a usability test was performed to identify the strong and weak spots and to verify if all functionalities implemented are intuitive and sufficient. The stages performed during the tests were:

1) The tester greets the participant and invites him to sit in front of the device.

2) The tester explains the motives for the test and also the research context. It is explained that will be made recordings during the test duration and that the objective of the test is to evaluate the interface and not the user.

3) It is presented to the participant the free informed consent term and it is asked to be red and signed by the participant.

4) The tester fills the demographic questionnaire making questions to the participant when necessary.

5) The tester then, delivers to the participant the task to be accomplished and the recording starts.

6) After the conclusion of the task it is delivered to the participant a satisfaction and acceptance questionnaire that must be filled by him

7) After the completion of this questionnaire the tester asks the participant if he has any doubts or commentaries and if there are it is noted and answered.

8) When it is over the tester thanks the presence and participation of the participant and makes final compliments.
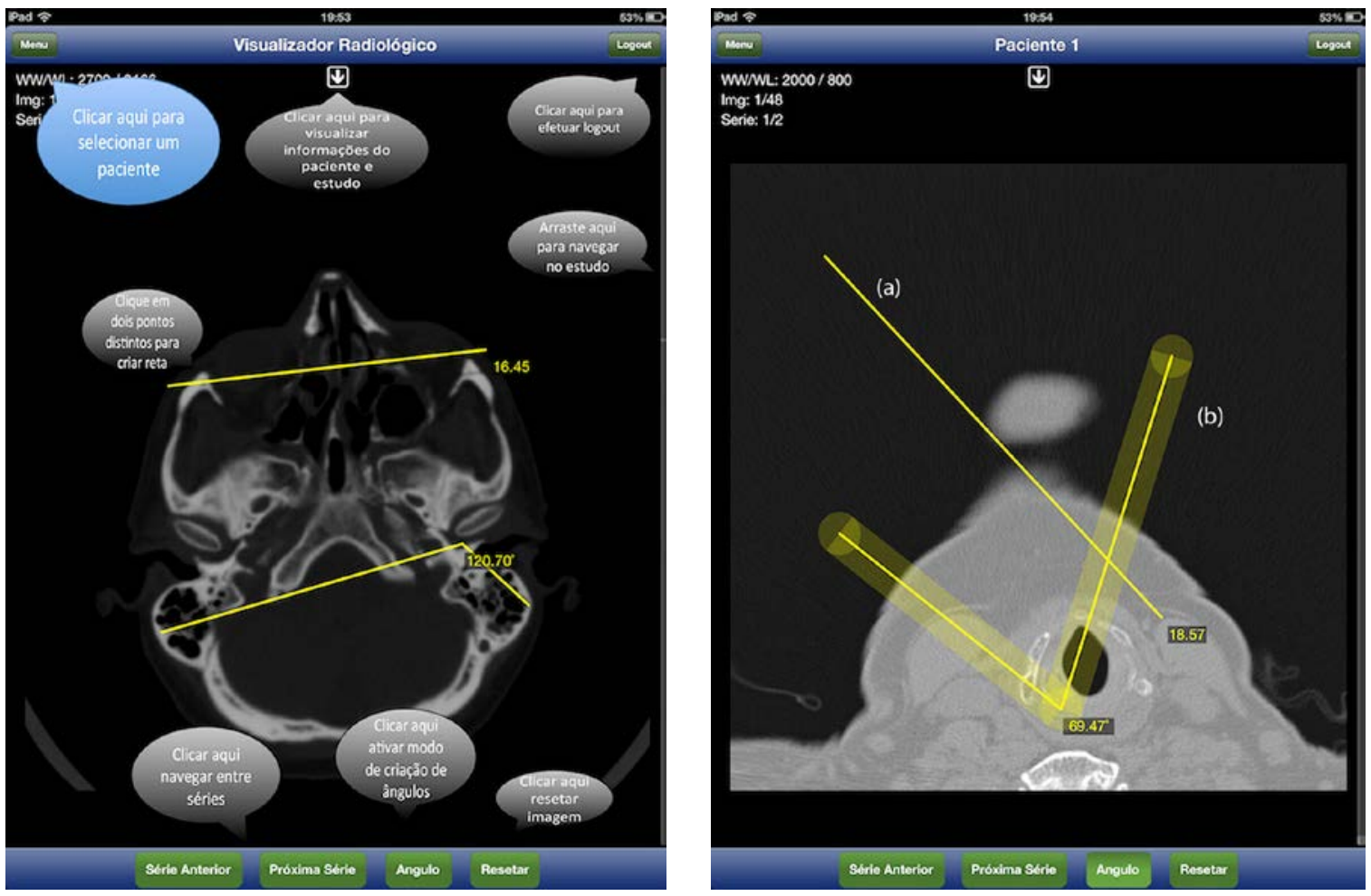

Fig.4. Left: Image visualization and analysis screen showing length and angle measurements and tooltips explaining the functionality of each screen tool. Right: The image measurement screen showing (a) an already performed length measure and (b) an angle measurement in the process of being executed.

After finishing the execution of the tasks the users answered a SUS satisfaction questionnaire. It has the objective to verify the satisfaction of the users in relation to the app.

It can be observed that the minimum score of the SUS questionnaire was $80 \%$ of satisfaction. It was expected a value above $75 \%$ so the result is considered really good, considering that the SUS has a minimum value of $0 \%$ and a maximum value of $100 \%$. Excellent values were also 
observed in the mean values for each question. With this data and verbal confirmations done at the end of the tests, it could be inferred that the app was well accepted by the participants.

It was reported by the majority of doctors that there is the necessity of more tools including:

-Point and region density (ROI);

-Measurement of small structures;

-Use of the scale in millimeters;

-Preferences for standard leveling and windowing of different tissues;

-MPR (Multi Planar Reconstruction);

-Dental reconstruction;

-At least 2 different visualizations simultaneously in the screen with scrolling in both of them;

So it could be inferred that there still is a need for improvements in order to all these functionalities be available. The main strong points shown by the participants where:

-Design has colors similar to the Report app (STT Mobile Version for the iPad), making it more easy for participants to get used to it;

- Login interface is similar to the Report App app (STT Mobile Version for the iPad) so is also familiar to the participants;

-Scroll bar works really well and was adopted almost instantly;

-Menu with the patient list has easy access and was considered practical;

-Image zoom with both fingers in a pinch movement was considered intuitive;

There were also weak spots raised by the participants:

-Handling the image (windowing and leveling) with 2 fingers simultaneously was not adopted rapidly;

-In ultrasound images the leveling and windowing must have better precision, it was hard for some participants to reach desired values;

-The was a difficulty in perform measurements in tiny body parts;

-Inversion of image scrolling, because now it is performed in ascending order, but all participants opted to start the scrolling from the top of the screen;

-In the patient list it was noted the need of date filters and requesting doctors associated with the DICOM studies.

-Measurements in centimeters must be converted to millimeters

\section{Conclusions and Future Works}

The work described in this paper had as main objective to identify critic questions related to the interface design of a teleradiology client on a tablet computer. To achieve this objective we performed a case study, creating user interfaces for a DICOM visualizer on iPad 2 that was incorporated into the STT following a pragmatic process of usability engineering. It was implemented also and functional application following a software engineering process.

This application was then evaluated by a series of usability tests with doctors of the unified health system in Santa Catarina (SUS). The application was considered practical and efficient and was well accepted by the participants of the study. We hope that this application will be used to improve the current process of generating reports in the Telemedicine System through portability, this way doctors can access these exams in an easier and faster way, without requiring a workstation or even being in a hospital.

In general, the usability tests results demonstrate a first indication that the application can meet the requirements of it's main objective, which is function as an auxiliary in the process of making and emitting a medical report for the STT system, through detailed analysis of DICOM images. According to the results the application was considered useful and objective.

The design of interfaces was considered very good by the participants, maintaining a pattern with the design of the already existing application for emission of medical reports called "STT 
Laudos". The access to images worked in an intuitive and fast way, it was praised by the participants mostly because the context where they are inserted depends on how fast they can access these images and make the report for them. The patient list was considered ideal, because it has almost all the tools necessary, there were some suggestions like the filter of patients by date.

For future works it is suggested the perfecting of the application implementing functionalities request by the test participants and integrating this application in the current context of telemedicine and Santa Catarina's heath system (SUS). There is also the suggestion to validate the application as a tool to help the creation of reports.

\section{References}

Choi, HJ, Lee, JH, and Kang, BS. Remote CT reading using an ultramobile PC and web-based remote viewing over a wireless network. J Telemed Telecare, 1 , 18 (Jan 2012), 26-31.

Kim, DK, Kim, EY, Yang, KH, Lee, CK, and Yoo, SK. A mobile teleradiology imaging system with JPEG2000 for an emergency care. J Digit Imaging, 4, 24 (Aug 2011), 709-718.

John, S, Poh, AC, Lim, TC, Chan, EH, and Chong, R le. The iPad tablet computer for mobile on-call radiology diagnosis? Auditing discrepancy in CT and MRI reporting. J Digit Imaging, 5, 25 (Oct 2012), 628-634.

Avitzur, O. In Practice: How Neurologists are Using the Newest Tablets - In and Out of the Clinic. Neurology Today, 10, 24 (Dec 2010), 22-24.

Weiss, FE, Jeudy, J, and Siegel, EL. TB or not TB? Answering an age old question using a modern device: The iPad. (2010), RSNA.

Choudhri, AF, Carr, TM, Ho, CP, Stone, JR, Gay, SB, and Lambert, DL. Handheld device review of abdominal CT for the evaluation of acute appendicitis. J Digit Imaging, 25, 4 (Aug 2012), 492-6.

Wasserman, A I. Software Engineering Issues for Mobile Application Development. (Santa Clara 2010), Proceedings of Workshop on Mobile Software Engineering, 397-400.

Chen, Chufeng, Abdelnour-Nocera, Jose, Wells, Stephen, and Pan, Nora. Usability Practice in Medical Imaging Application Development. Lecture Notes in Computer Science, 5889 (2009), 405-415.

Drnasin, Ivan and Grgic, Mislav. "The use of mobile phones in radiology," ELMAR, 2010 PROCEEDINGS, Zadar, 2010, pp. 17-21.

Cannella, V.; Orazio, G.; Pirrone, R.; Vitabile, S. GUI Usability in Medical Imaging. CISIS 2009: 778-782

ISO. International Organization for Standardization. ISO 9241 Requisitos Ergonômicos para Trabalho de Escritórios com Computadores Parte 11 - Orientações sobre Usabilidade, 2002.

Chan,. Usability evaluation of order sets in a computerized provider order entry system. BMJ Qual Saf. v. 20, n. 11, p. 932-40, 2011.

Yen, P..Y. Health Information Tecnology Usability Evaluation: Methods, Models and Measures. Ann Arbor: ProQuest, UMI Dissertation Publishing, 162 p., 2011.

Siau, K. Mobile communications and mobile services. International Journal of Mobile Communications. v. 1, n. 1-2, p. 3-14, 2004.

STT. Disponível em: https://telemedicina.saude.sc.gov.br/rctm/. Acessado em: 02 mar. 2016.

Nielsen, Jakob. "Enhancing the explanatory power of usability heuristics."Proceedings of the SIGCHI conference on Human Factors in Computing Systems. ACM, 1994. 